\section{Neurophysiologic Studies in Metallosis Oculi}

\author{
Donald F Farrell* \\ Neurology, EEG and Clinical Neurophysiology, University of Washington \\ School of Medicine, Seattle, WA, USA
}

\begin{abstract}
Metallosis oculi remains a cause of retinal damage in spite of attempts to prevent this disorder by the use of protective goggles. Ultrasound biomicroscopy is of value in the diagnosis of a retained metal fragment in ocular metallosis, but neurophysiologic tests are required to confirm the diagnosis and to follow the course of the disease. The objective of this manuscript is to identify the most sensitive neurophysiologic test to diagnose the presence of a retained metal fragment.
\end{abstract}

Aim: To show that the Pattern reversal Electroretinogram (PERG) is more sensitive in establishing a diagnosis of metallosis oculi than the corneal full-field Electroretinogram (ERG). The ERG is however more useful in following the case than the PREG which may be absent.

Methods and material: Corneal full-field Electroretinograms (ERGs), Pattern reversal Electroretinograms (PERGs), were carried out on referred patients in a tertiary neurophysiologic laboratory. There were ten cases, all males, with a mean age of 42 years. The retained metal fragments were present from months to years with the longest being about 40 years. These studies were accomplished in order to determine which individuals would benefit from the surgical removal of the retained metal fragment.

Results: The PERG is the most sensitive test in establishing a diagnosis of a retained metal fragment, however, it may not be optimal for following the course of the illness.

Conclusion: The PERG is the most sensitive test currently available to establish a diagnosis of a retained metal fragment, but may not be of value if the patient has either glaucoma or the PERG shows an absent response. Other tests such as the ERG or PREP may be of greater value in following the course of the illness.

Keywords: Corneal full-field electroretinogram; Metallosis; Pattern reversal electroretinography; Pattern Reversal Evoked Potential (PREPs); Visual loss

*Corresponding author: Donald F Farrell, Neurology, EEG and Clinical Neurophysiology, University of Washington School of Medicine, Seattle, WA 98195, USA, Tel: +1 2064374026; E-mail: donf@u.washington.edu

Citation: Farrell DF (2015) Neurophysiologic Studies in Metallosis Oculi. J Ophthalmic Clin Res 2: 015.

Received: August 05, 2015; Accepted: October 27, 2015; Published: November 10, 2015

\section{Introduction}

Ocular metalloses has been known to exist since the 1860s [1]. However, it probably existed since the iron age, but this disorder increased in frequency with the industrial revolution and modern wars. Protective eye wear has reduced the incidence, but cases continue to be seen on a regular basis. Acutely, it is important to identify and remove any metal fragment so as to prevent progressive damage to the eye. Ocular ultrasound biomicroscopy $[2,3]$, appears to be a reliable method to identify acute lesions so that the fragment can be located and removal can be completed. If however, the affected individual does not seek medical attention at the time of the accident, the chance of a retained metal fragment causing retinal damage increases and it may be that this individual seeks medical attention at a later date. It is important to recognize the presence of retained metal fragments as progressive loss of vision occurs in many cases and it is important to remove the retained metal fragment to prevent progressive visual loss. The most common causes are iron followed by copper. These metals are toxic when traumatically introduced into the eye. In experimental animals iron introduced into the orbit was absorbed into the eye and caused to ocular metallosis.

In many cases the eye attempts to isolate the introduced metal fragment by encapsulation [4], but progressive visual loss may still occur. Other cases lead to siderosis or generalized rust throughout the eye. For many years, beginning in the 1950s [5], corneal full-field electroretinography has been the test of choice in determining retinal damage, but more recently Pattern reversal Electroretinography (PERG) has been found to be more sensitive in detecting early changes. PERG measures the function of the ganglion cell layer of the retina [6], a group of neurons sensitive to the presence of metal in the eye. The ganglion cell layer is responsible for the formation of the optic nerve and the first change in the Pattern Reversal Evoked Potential (PREPs) is a reduction in amplitude of the P100 when ganglion cells are lost. This may progress to a complete loss of the p100 in severe cases.

\section{Subjects and Methods}

Ten cases of ocular metalloses were studied between the years 1983 and 2003. All cases were male with a mean age of 42 years (range 14-65). The intra-ocular metal was either iron or steel in 9 cases and copper in 1 case. The ocular findings included encapsulated foreign body in 5 cases and siderosis in 5 cases. The foreign body had been present for months to many years. Four cases underwent cataract removal with artificial lens replacement and one case had a corneal transplant. Visual acuity varied greatly from individual to individual. One individual had symmetric VA at 20/15 OU, the remainder had asymmetric responses with the best being 20/25 OS and 20/40 OD to the worst with $20 / 25$ OS and counting fingers at 1 and $1 / 2$ feet OD.

The period of time in which this study was accomplished covered a period of time when a corneal full-field electroretinogram was the principle diagnostic test and ended with the principle test being the Pattern reversal Electroretinogram (PERG), a test that turns out to be more sensitive. Six cases underwent testing with corneal full-field 
ERGs, three cases had both corneal full-field ERGs (ERG) and pattern reversal electroretinography. The ERG is carried out using a Burien-Allen corneal electrode to record responses (Figure 1-Burien-Allen corneal electrode). If a given patient cannot tolerate this electrode or if they have nystagmus a gold leaf electrode can be used instead. The gold leaf electrode records the responses without loss of amplitude or latency. A series of tests in both the dark adapted state with blue flash, red flash and white flash, measuring predominantly the rod function and progressing to cone function with $30 \mathrm{~Hz}$ white flicker then in the light adapted state, white flash, yellow-red and blue-green flash to measure cone function or subsets of cone function [7].

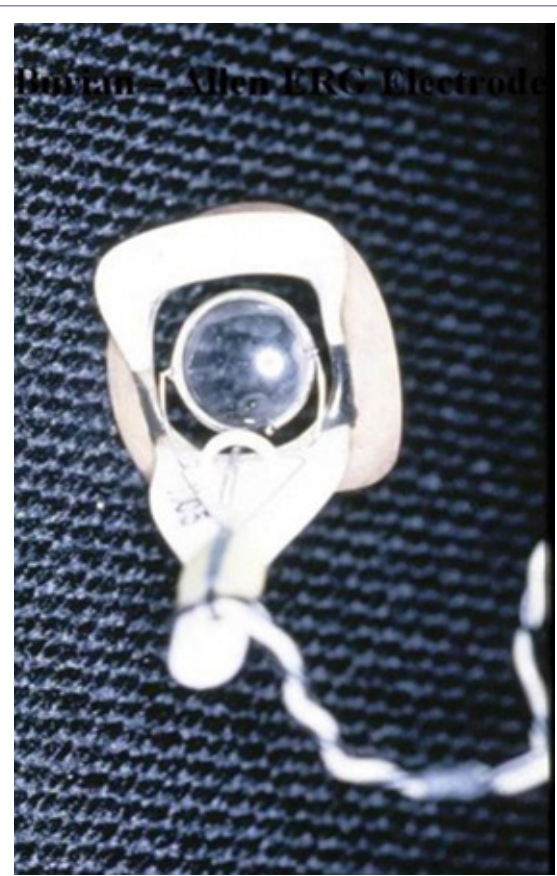

Figure 1: Photograph of Burien-Allen corneal electrode which is used to collect data for both corneal full-field ERGs and PERGs.

In actuality, only the blue flash in the dark adapted state is a pure rod test, cone oscillations are seen with red flash and a double a wave with a1 being of cone origin and a2 being of rod origin, the B wave is a mixture of the two to white flash. PERG testing uses the same recording electrode, but uses a checkerboard pattern with 30 minute visual angle as the stimulus. Six cases underwent PERG testing while three had both ERG and PERG testing.

Six cases underwent Pattern Reversal visual Evoked Potentials (PREPs), with one being symmetric and normal; the remainder showed various abnormalities in the affected eye with reduction in amplitude of the p100 being the most common abnormality, two of the abnormal cases showed no response at all. Abnormalities in the PERG include an amplitude difference between the eyes of 50 percent or greater. One case had simultaneous metallosis in one eye and glaucoma in both, with both eyes having abnormal PERGs. An Electro-Oculogram (EOG) was accomplished in 2 cases. A light peak to dark trough ratio method was used to establish whether the result was normal or abnormal. One was normal in both the normal and affected eye (2.41 OD and 2.93 OS) and one showed an abnormal response in the affected eye (1.36 OS and 2.25 OD). A normal value should be greater than $1.72 \mathrm{OU}$.

\section{Results}

For many years the corneal full-field electroretinogram has been the diagnostic neurophysiologic test of choice in the diagnoses of a retained metal foreign body in the eye. Because of the potential for progressive visual loss it has been imperative to identify those individuals at risk for progression. In this current series, seven cases underwent corneal full-field flash electroretinograms. One had a perfectly normal study, see (Figure 2, normal corneal full-field ERG). This same individual had an absent right pattern reversal electroretinogram (Figure 3, absent PERG, OD). The remaining cases showed asymmetric changes in the ERG with the abnormalities ranging from mild to no recordable responses (see figure 4, abnormal corneal full-field ERG). Six individuals were studied with PERG and all showed asymmetric abnormalities with the most common finding of an absent response. One individual had bilateral absent PERGs and was noted to have not only a question of a retained metal fragment, but bilateral glaucoma. Six cases underwent Pattern Reversal Evoked Potentials (PREPs) either as part of the PERG study or as a standalone test (Figure 5, abnormal PERG and PREP in ocular metallosis). Abnormalities in the PREP included either decrease in the amplitude of the p100 or a prolonged latency (implicit time) of the p100, or both. The changes in PREPs are thought to be the result of damage to the ganglion cell layer. Two cases had an electro-oculogram which measures retinal pigment epithelial function, with one case being normal and one abnormal.

\section{Discussion}

Surgical removal of the metal fragment as soon as possible is the best possible therapy to prevent the late and progressive changes. In recent years, ultrasound biomicroscopy [2,3], has been used to identify and localize retained metal fragments in the eye. In each instance that this procedure has been used for the successful removal of the retained fragment, it has led to the prevention of progression of visual loss. The number of these cases is small so it is not possible at this time to know if this test will miss cases. In a series of 48 cases [8], that were studied, forty five had the metal fragment removed before ocular metallosis could develop. Three cases presented late and already showed signs and symptoms of Ocular metallosis.

Pattern reversal Electroretinograms (PERGs) are more sensitive than corneal full-field flash Electroretinograms (ERGs) in the detection of retained metal fragments in the eye. The most common finding is an absent PERG in the affected eye.

By the time the ERG is abnormal significant damage has already been done to the retina. The PERG becomes abnormal to absent before any changes occur in the ERG itself, making it the superior test. One major complicating factor may be the co-existence of glaucoma which also affects the PERG [6,9], in that with glaucoma both eyes are likely to be affected. Retained metal fragments may also cause glaucoma, but that should only occur in the traumatized eye, not both.

Direct fundoscopic examination is likely to suggest the presence of ocular metallosis. Two major changes are likely, 1) the presence of an encapsulated fragment or 2) siderosis, or a generalized staining of the ocular contents.

Intra-ocular pressure should then be measured and if the unaffected eye is normal, then a PERG should be obtained to confirm the existence of a retained metal fragment. Once confirmed the retained metal fragment should be removed to prevent any further visual loss. The PERG response may be absent early in the course so 


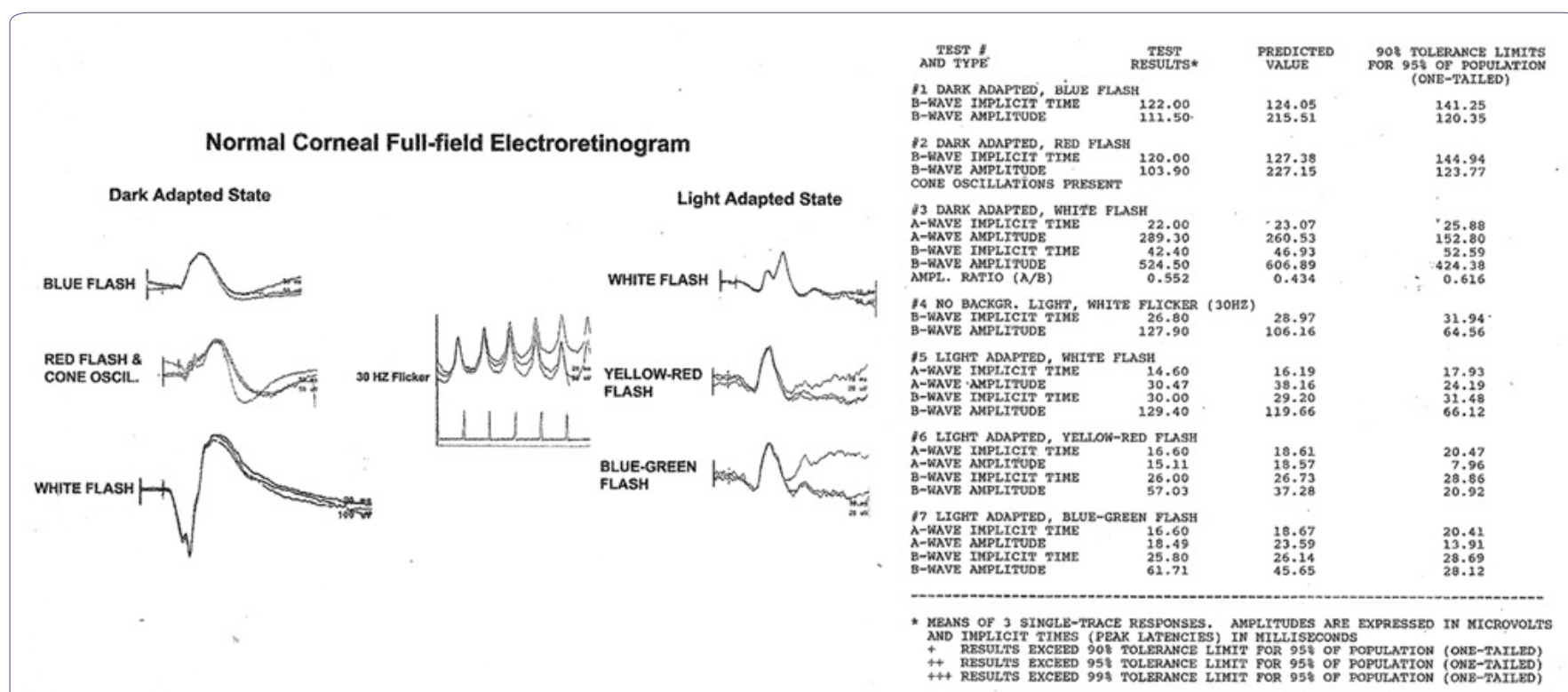

Figure 2: Normal corneal full-field ERG responses with quantitative implicit times and amplitudes to the 7 test conditions in a patient that had a retained metal fragment, but had an abnormal PERG.

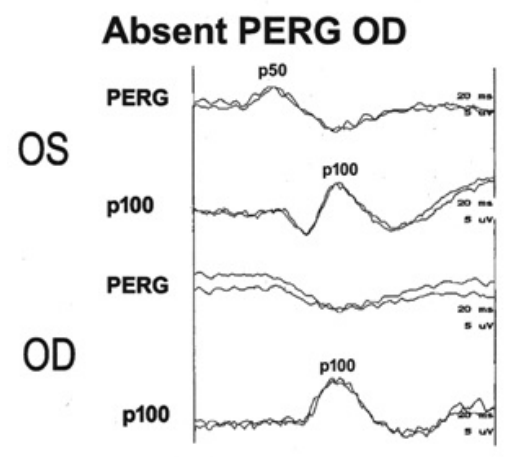

Figure 3: Abnormal PERG showing absent response OD, OS shows a normal p50. These findings are suggestive of progressive changes secondary to ocular metallosis, $\mathrm{OD}$.

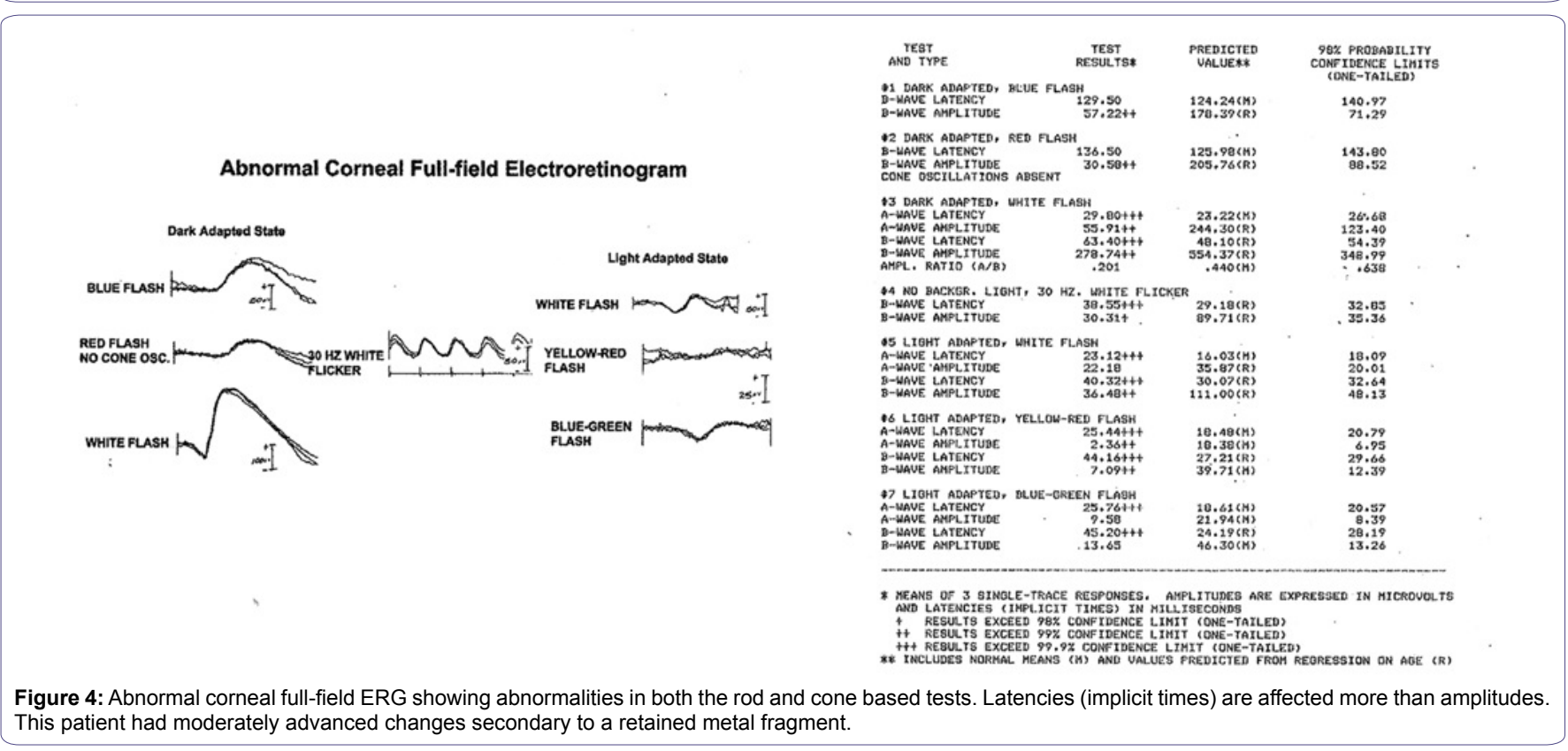




\section{Abnormal PERG and PREP in Ocular Metallosis (OS)}

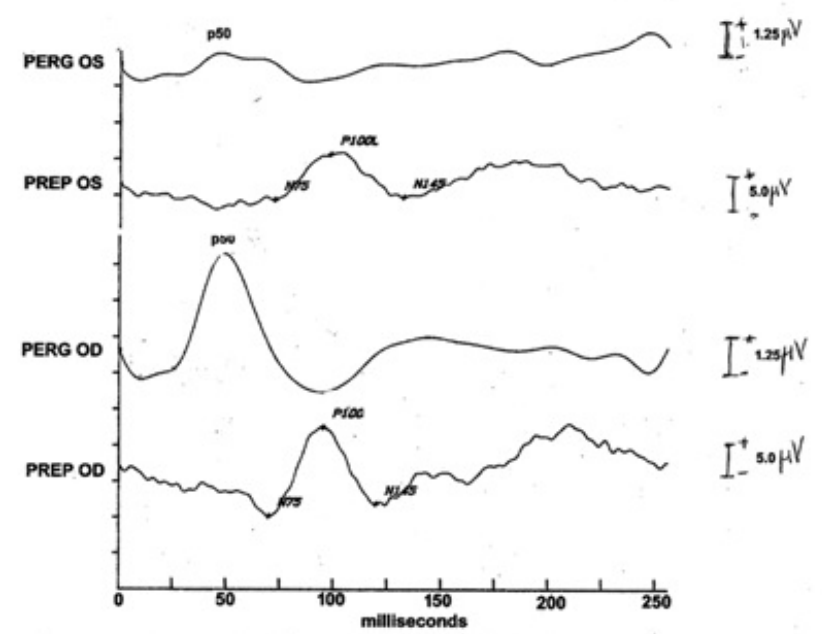

Figure 5: Abnormal PERG and PREP OS. OD shows normal responses. This patient had a retained metal fragment in his eye. The ganglion layer of the retina had enough damage to affect the PREP.

may not be of great value in following cases to determine if progression is occurring. If progression is suspected then a corneal full-field flash ERG may be used to follow the course of the illness. One additional test may be of value in following progression, but it has not been studied. This test is the Pattern Reversal Evoked Potential (PREPs). Five cases in this current study showed abnormal PREPs. In all cases the amplitude of the p100 was reduced reflecting the involvement of the ganglion cell layer, the origin of the optic nerve in the eye.

The proper management of a traumatic retained metal fragment may require the use of several tests. Ultrasound biomicroscopy appears to be an excellent method for the identification and localization of acute metal fragment within the first few days of injury. The PERG is sensitive in the identification of retained metal fragments, but when the response is absent may be of no value in following those cases that may later show progression. The corneal full-field flash ERG still has some value in following the course of the disorder and PREPs may also be of value in following a given case.

\section{References}

1. vonGraefe A (1860) Cataracta traumatica und chronische Choroiditis durch einen fremden Körper in der Linse bedingt, von Graefe's Arch. Ophthmal 6: 134-139.

2. Chobeaux N, Maux R, Colin J, Korobelnik JF (2003) [Delayed discovery of a metallic intraocular foreign body: diagnostic and therapeutic stakes]. J FrOphthmol 7: 730-733.

3. Ambartsumian AR (2011) Potential of ultrasound biomicroscopy in diagnosis of ocular trauma with intraocular metallic foreign bodies. Vestn Oftalmol 4: 29-33.

4. Roper-Hall MJ (1954) Review of 555 cases of intraocular foreign body with special reference to prognosist ${ }^{\star}$. Br J Ophthmal 38: 65-99.

5. Karpe G (1957) Das Electroretinogram bei Siderosis bulbi. Bibl Ophthmal 48: 182-190.

6. Ventura LM, Golubev I, Feuer WJ, Porciatti V (2013) Pattern electroretinogram progression in glaucoma suspects. J Glaucoma 22: 219-225.

7. Farrell DF (2009) Unilateral retinitis pigmentosa and cone-rod dystrophy. Clin Ophthalmol 3: 263-279.

8. Gracner B, Pahor D, Gracner T (2003) [Siderosisbulbi. Is it still a problem?]. Ophthalmologe 100: 1045-1048.

9. Bach M, Unsoeld AS, Philippin H, Staubach F, Maier P, et al. (2006) Pattern ERG as an early glaucoma indicator in ocular hypertension: a long-term, prospective study. Invest Ophthalmol Vis Sci 47: 4884-4887. 